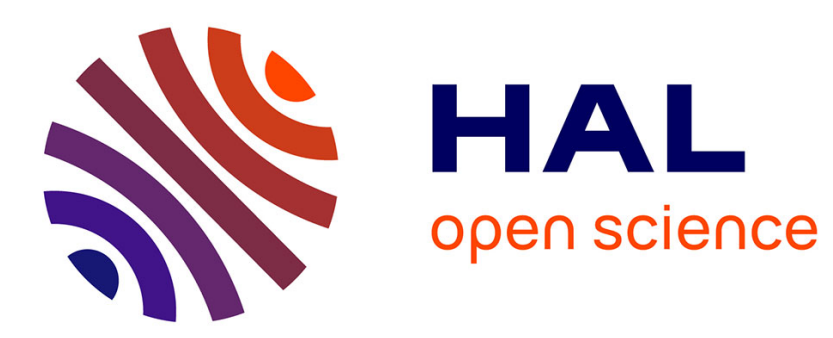

\title{
Quel " monde du contact " ? Pour une histoire sociale de l'Algérie pendant la période coloniale
}

\author{
Emmanuel Blanchard, Sylvie Thénault
}

\section{To cite this version:}

Emmanuel Blanchard, Sylvie Thénault. Quel "monde du contact"? Pour une histoire sociale de l'Algérie pendant la période coloniale. Le Mouvement social, 2011, 236 (3), pp.3. 10.3917/lms.236.0003 . hal-02355618

\section{HAL Id: hal-02355618 \\ https://hal.science/hal-02355618}

Submitted on 12 Nov 2019

HAL is a multi-disciplinary open access archive for the deposit and dissemination of scientific research documents, whether they are published or not. The documents may come from teaching and research institutions in France or abroad, or from public or private research centers.
L'archive ouverte pluridisciplinaire HAL, est destinée au dépôt et à la diffusion de documents scientifiques de niveau recherche, publiés ou non, émanant des établissements d'enseignement et de recherche français ou étrangers, des laboratoires publics ou privés. 


\title{
Éditorial
}

\section{QUEL « MONDE DU CONTACT »? POUR UNE HISTOIRE SOCIALE DE L'ALGÉRIE PENDANT LA PÉRIODE COLONIALE}

\author{
Emmanuel Blanchard et Sylvie Thénault
}

La Découverte | « Le Mouvement Social »

$2011 / 3 n^{\circ} 236$ | pages 3 à 7

ISSN 0027-2671

ISBN 9782707169655

Article disponible en ligne à l'adresse :

https://www.cairn.info/revue-le-mouvement-social-2011-3-page-3.htm

Distribution électronique Cairn.info pour La Découverte.

(C) La Découverte. Tous droits réservés pour tous pays.

La reproduction ou représentation de cet article, notamment par photocopie, n'est autorisée que dans les limites des conditions générales d'utilisation du site ou, le cas échéant, des conditions générales de la licence souscrite par votre établissement. Toute autre reproduction ou représentation, en tout ou partie, sous quelque forme et de quelque manière que ce soit, est interdite sauf accord préalable et écrit de l'éditeur, en dehors des cas prévus par la législation en vigueur en France. Il est précisé que son stockage dans une base de données est également interdit. 


\title{
É D I \\ Quel « monde du contact » ? Pour une histoire sociale de l'Algérie pendant la période coloniale
}

\author{
par Emmanuel Blanchard et Sylvie Thénault ${ }^{*}$
}

Sans qu'il soit toujours circonscrit, défini, ni même qualifié en ces termes, le «monde du contact " est une notion qui irrigue l'historiographie française de l'Algérie coloniale. Il est au cœur d'un schéma narratif focalisé sur le politique et dont la chronologie repose sur la litanie des réformes inabouties : les " occasions perdues " ${ }^{1}$. L'itinéraire d'un Ferhat Abbas, le pharmacien de Sétif, porte-parole des revendications d'égalité en faveur des colonisés d'Algérie, en est devenu le symbole. Réputé pour son scepticisme au sujet de l'existence d'une nation algérienne dans l'entre-deux-guerres, il tenta une proposition médiane pendant la Seconde Guerre mondiale en imaginant une Algérie aux liens assouplis avec la France, avant de prendre la tête du premier Gouvernement provisoire de la République algérienne (GPRA) formé par le Front de libération nationale (FLN) en pleine guerre d'indépendance. À son image, le «monde du contact " raconterait l'histoire d'un espoir déçu par l'incapacité française à corriger, par la réforme, les injustices criantes de la société coloniale. D'un point de vue algérien, les acteurs de ce "monde " auraient incarné l'espoir d'un pluralisme politique, réduit à néant par le FLN qui s'imposa, violemment, comme force exclusive de la lutte pour l'indépendance ${ }^{2}$.

Suivant une version plus critique de l'idée que le réformisme colonial aurait pu tracer d'autres chemins, et notamment éviter une guerre de décolonisation qui l'aurait éradiqué, le "monde du contact " ne serait qu'une illusion : ce " monde " n'avait qu'une surface sociale étroite et il était de consistance fragile, en regard de la discrimination séparant les habitants de l'Algérie coloniale en deux groupes. Statuts et droits les placèrent de part et d'autre d'une frontière que renforçaient les inégalités socio-économiques, ainsi qu'une répartition ségrégative entre villes et campagnes - les colons furent d'emblée urbains tandis que les Algériens

\footnotetext{
* Respectivement maittre de conférences à l'UVSQ, Centre de recherches sociologiques sur le droit et les institutions pénales (CESDIP, UMR 8183), et chargée de recherches au CNRS, Centre d'histoire sociale du XX siècle (CHS, UMR 8058).

1. Charles-André Julien est le représentant éminent de cette interprétation, reprise par son élève Charles-Robert Ageron. Il conclut son ouvrage L'Afrique du Nord en marche sur « la politique des occasions perdues ». Le livre parut pour la première fois chez Julliard en 1952. "Occasions manquées" est toutefois l'expression la plus répandue.

2. M. Rahal, Ali Boumendjel. Une affaire française, une histoire algérienne, Paris, Les Belles Lettres, 2010.
} 
restèrent majoritairement ruraux $^{3}$. La taxinomie contemporaine révèle la " coloration raciale » ${ }^{4}$, typiquement coloniale, de cette division. Elle mêlait lignage et religion : "Européens » et " Musulmans » désignaient les uns et les autres.

La notion de " monde du contact » est ainsi arrimée à un questionnement politique sur la nature des rapports sociaux dans l'Algérie coloniale et sur leurs potentialités : elle oppose l'existence de relations sincèrement amicales, voire fraternelles, entre " colons " et "indigènes ", à la focalisation sur la séparation des groupes, la force et la domination. Cet arrimage est symptomatique d'une historiographie guidée par la guerre d'indépendance et la violence, au détriment d'une attention davantage tournée vers les moments ordinaires de la longue durée coloniale et ses acteurs les plus anonymes. L'existence de contacts, pourtant, n'est rien moins qu'une évidence. Cent trente ans d'une tutelle française concrétisée par un peuplement venu d'Europe atteignant le million en fin de période, ne pouvaient que reconfigurer les rapports sociaux et les dispositifs de pouvoir, même vernaculaires.

Dans le cas de l'Algérie, cependant, la phase finale de la période coloniale et ses violences sont toujours en ligne de mire des interrogations sur les relations entre groupes, les rapports interindividuels, les emprunts et les hybridations de pratiques voire les métissages. De ce fait, l'historiographie de l'Algérie coloniale a suivi une trajectoire spécifique. Elle est restée à l'écart d'approches et d'écritures visant à réinscrire le " moment colonial " dans des historicités vernaculaires de longue durée ${ }^{5}$. Ce cloisonnement est également marquant par rapport aux subaltern studies qui, depuis trente ans, ont renouvelé l'histoire sociale des populations colonisées. Celles-ci ont peu touché l'historiographie de l'Afrique du Nord ${ }^{6}$. De ce point de vue, les travaux de Fanny Colonna, au croisement de la sociologie et de l'histoire, avaient été précurseurs. Ils occupent aussi une place de choix dans un ouvrage programmatique des études impériales ${ }^{7}$.

3. En 1886, 68 \% des Européens étaient urbains. Le pourcentage s'élève à $88 \%$ en 1954. Chez les Algériens, ils ne sont que $24 \%$ à vivre en ville à cette date. Voir K. Kateb, Européens, "indigènes » et juifs en Algérie (1830-1962). Représentations et réalités des populations, Paris, INED, 2001.

4. Selon l'expression de G. BaLAndier dans « La situation coloniale : approche théorique ", Cahiers internationaux de sociologie, vol. 11, 1951, p. 73.

5. R. Bertrand, "Les sciences coloniales et le "moment colonial" : de la problématique de la domination coloniale à celle de l'hégémonie impériale ", Questions de Recherche, n ${ }^{\circ} 18$, 2006, p. $1-41$.

6. F. Colonna, «The Nation's 'Unknowing Other': Three Intellectuals and the Culture(s) of Being Algerian, or the Impossibility of Subaltern Studies in Algeria ", The Journal of North African Studies, vol. 8, n 1, 2003, p. 155-170 ; D. DJerbal, "Critique de la subalternité ", Rue Descartes, ${ }^{\circ}$ 58, 2007, p. 84-101.

7. F. Colonna, "Educating Conformity in French Colonial Algeria » in F. Cooper \& A. Stoler (eds.), Tensions of Empire. Colonial Cultures in a Bourgeois World, Berkeley, University of California Press, p. 346-370. Ce chapitre est une traduction d'extraits de F. Colonna, Instituteurs algériens 1883-1939, Paris, Presses de la FNSP, 1975. 
L'histoire sociale de l'Algérie coloniale est riche de références et d'auteurs. Mais elle relève surtout d'une histoire sociale du politique dont Omar Carlier, Mohammed Harbi, Gilbert Meynier ou encore René Gallissot ont montré la fécondité pour l'étude du nationalisme algérien et du mouvement ouvrier. Dans le contexte d'une historiographie à fort signifiant politique et marquée par une tentation téléologique, l'attention se porte difficilement sur les acculturations ou les reconfigurations. Un tel contexte délégitime l'analyse des groupes sociaux intermédiaires, engloutis dans les rétrécissements identitaires des dernières années de la colonisation. L'attention portée à ces groupes est en effet comprise comme une tentative de réécriture de l'histoire de l'Algérie, comme une façon de signifier qu'un autre destin politique aurait été possible. De fait, le "monde du contact» imprègne l'historiographie mais ses contours n'ont jamais été précisément tracés. Les travaux manquent sur ce point. Un " minuscule creuset ", " capable de créer un monde de relations réelles entre les communautés ", propose Annie Rey-Goldzeiguer dans un effort rare de définition de la notion ${ }^{8}$. Étaient susceptibles d'en être, parmi les colonisés, les « agents de l'autorité », les « intellectuels ", les «politiques formés par la France », les " jeunes des écoles", les " évolués" dans le " vocabulaire algérien "?

Le premier objectif des articles rassemblés dans ce numéro est d'étayer la connaissance de ce "monde du contact ». Ils en approfondissent l'étude, en explorant un milieu socio-professionnel (le journalisme), des espaces (la presse, le mouvement ouvrier, la ville) et un moment (le Front populaire) propices au franchissement de la frontière coloniale. Mais il s'agit aussi d'interroger les fondements implicites de la notion. Car si l'existence de contacts est une évidence, elle ne préjuge en rien des trajectoires politiques et des historicités plurielles, placées au centre d'analyses récentes ${ }^{10}$. Pourquoi les contacts auraient-ils produit échanges et compréhension mutuels ? Le cas des journalistes est frappant à cet égard ${ }^{11}$. Comment ignorer, aussi, que les élites des rangs colonisés, même francophones et en relation avec leurs homologues européennes, animaient parallèlement des espaces de sociabilité, dans un esprit de ressourcement collectif fermé, si ce n'est opposé, au colonisateur ? L'activité foisonnante des ishalistes à Alger dans les années 1930, sous la houlette des notables locaux, tempère ainsi, par contraste, l'enthousiasme du Front populaire ${ }^{12}$; moment éphémère, celui-ci reste une exception. Mis en regard l'un de l'autre, les articles d'Afaf Zekkour et de Claire Marynower témoignent de transferts et d'un partage de répertoires : les islahistes investissent la forme associative et la symbolique musulmane marque les gestes des membres du Front

8. A. Rey-Goldzeiguer, Aux origines de la guerre d'Algérie, 1940-1945: de Mers-el-Kébir aux massacres du Nord-Constantinois, Paris, La Découverte, 2002, p. 76.

9. Ibid.

10. G. Lydon, On Trans-Saharan Trails: Islamic Law, Trade Networks, and Cross-Cultural Exchange in Nineteenth-Century Western Africa, Cambridge, Cambridge University Press, 2009.

11. Voir l'article de Philipp Zessin.

12. Il faudrait lire en parallèle les articles d'Afaf Zekkour et de Claire Marynower. 
populaire en Algérie. Une fois encore, cependant, pourquoi ces transferts et ce partage auraient-ils servi de passerelles ? L'emprise des assignations différentielles ne disparut pas dans les sociabilités ni dans les pratiques transgressives de la frontière coloniale.

La notion de "monde du contact ", enfin, perd de sa pertinence dès que le regard se déplace de la ville vers la campagne, dans l'un de ces isolats traditionnellement présentés comme ayant vécu à l'écart de la colonisation. Le renversement de perspective est édifiant : pour les Béni-Boudouane, la colonisation française ne s'incarna pas, sauf tardivement, dans la présence physique d'Européens ${ }^{13}$. Aucun " monde du contact " possible, par conséquent. La notion de " contact», pourtant, a ici aussi un sens. Mais ce « contact» ne mit pas « Européens » et « Musulmans » en présence. Il passa essentiellement par l'intermédiaire d'auxiliaires issus de la société locale, assurant l'interface avec les autorités coloniales. L'exemple démontre la nécessité de dépasser les questionnements sur la sous-administration dans l'évaluation du poids de la domination : la force du pouvoir ne se mesure pas à sa proximité. Ce sont aussi des relations à distance qui définissent le gouvernement colonial. La bureaucratisation et l'anonymisation des relations sociales ne sont certes pas aussi poussées que dans d'autres régions, mais elles s'incarnent par exemple dans le droit forestier ou la figure du garde-champêtre. À la fois proche des habitants et relié à un pouvoir lointain, inconnu, il était porteur d'une domination connue de chacun.

Ce dossier relève ainsi d'une tentative d'analyse de la consistance des rapports sociaux entre acteurs assignés à des appartenances différentielles par la situation coloniale ${ }^{14}$. Dans un contexte historiographique en plein renouvellement, marqué notamment par l'éclosion de travaux sur le XIX siècle ${ }^{15}$, ces articles sont surtout centrés sur la période de l'entre-deux guerres. Ils questionnent, au travers d'études empiriques, la notion de " monde du contact ", pour en évacuer la charge politique et téléologique, pour redonner toute leur place aux concepts et aux outils ordinaires d'une histoire sociale des segmentations urbaines, des groupes professionnels, des relations partisanes, des sociabilités militantes et religieuses. Leurs auteurs jettent sur cette histoire des regards différents, produits de la recherche arabophone en Algérie et de l'internationalisation croissante des études sur le pays ${ }^{16}$. Cette dernière prospère sur le " terrain algé-

13. Renversement que met en œuvre l'article de Giulia Fabbiano.

14. Ces articles sont issus de communications dans le cadre du séminaire "Populations et rapports sociaux en situation coloniale : le cas algérien (XIX'-XXe siècles) » co-organisé depuis trois ans par le CESDIP et le CHS.

15. Voir le récent dossier "L'Algérie au XIX siècle » de la Revue d'histoire du XIX siècle, en particulier l'introduction historiographique des trois coordinatrices de ce numéro : H. BLAIS, C. FREDJ, E. SAADA, «Un long moment colonial : pour une histoire de l'Algérie au XIX siècle ", Revue d'histoire du XIX" siècle, nº41, 2010, p. 7-24.

16. La translittération de l'arabe vers l'alphabet latin n'a pas été uniformisée. Plusieurs orthographes d'un même nom peuvent cohabiter, ces variations témoignant aussi de choix de sources et de regards différents portés sur ces documents. 
rien pour la recherche ${ }^{17}$, ouvert depuis le retour relatif de la sécurité dans le pays. Certes, les archives conservées en Algérie relèvent surtout d'une production administrative ; ce sont celles que les Français ont laissées à leur départ. Autour de ce noyau dur, cependant, les témoignages et les archives privées, que les acteurs et leurs familles ont conservés par devers eux, contribuent à multiplier les points d'entrée dans la complexité du moment colonial.

Avec ces études en forme de retour sur le " monde du contact ", ce dossier vise à décloisonner l'histoire de l'Algérie, trop souvent enfermée dans une trajectoire spécifique qui l'a laissée en marge de l'histoire impériale. Ainsi, la construction coloniale des Béni-Boudouane ne déroge pas à la règle générale selon laquelle le colonisateur solidifia les segments sociaux locaux pour fonder son organisation administrative et son État. La démarche prônée se veut toutefois attentive aux spécificités d'une situation coloniale algérienne qui à, bien des égards, fut exceptionnelle ${ }^{18}$. Au-delà même de la violence de la prise de possession du pays, perdurant dans le temps long de la colonisation ${ }^{19}$, d'autres caractéristiques singulières de ce long moment colonial peuvent être relevées. Jocelyne Dakhlia, par exemple, souligne que 1830 ne fut pas le moment de la "rencontre " entre Européens et Maghrébins, au contraire d'autres cas dans lesquels la " rencontre impériale " fut celle des " premiers contacts ${ }^{20}$; mondes occidental et arabo-musulman étaient en relation de longue date ${ }^{21}$. La démarche esquissée dans ce dossier se voudrait ligne de conduite à l'avenir : s'affranchir de tout exceptionnalisme méthodologique, afin de rendre compte des régularités de la situation coloniale en Algérie ; mais sans perdre de vue les singularités de cette situation, ni celles de son historiographie propre.

17. Ont été pionniers en ce sens R. Bader, A. Kudo, D. Guignard, "Un terrain algérien pour la recherche ", Vingtième Siècle. Revue d'histoire, n77, 2003, p. 110-112. La démarche est aujourd'hui évidente: A. Lacroix, C. Marynower, H. Vermeren, « Retour sur les archives algériennes ", Vingtième Siècle. Revue d'histoire, n¹10, 2011, p. 147-149.

18. Pour une analyse de l'Algérie colonisée comme " cas extrême ", M. Connelly, L'arme secrète du FLN. Comment de Gaulle a perdu la guerre d'Algérie, Paris, Payot, 2011, p. 18.

19. R. Branche, L'embuscade de Palestro. Algérie 1956, Paris, A. Colin, 2010 ; B. Brower, A Desert Named Peace: The Violence of France's Empire in the Algerian Sahara, 1844-1902, New York, Columbia University Press, 2009 ; J.-P. Peyroulou, Guelma 1945. Une subversion française dans l'Algérie coloniale, Paris, La Découverte, 2009.

20. Comme dans le cas euro-asiatique : R. Bertrand, «Rencontres impériales. L'histoire connectée et les relations euro-asiatiques ", Revue d'histoire moderne et contemporaine, vol. 54, n% bis, supplément 2007, p. 69-89.

21. J. Dakhlia, Islamicités, Paris, PUF, 2005, p. 70. 